\section{A123 GALACTOSE MODIFIED INKT CELL AGONISTS STABILISED BY A NOVEL STRUCTURAL MODIFICATION OF CD1D LEAD TO MARKED TH1 POLARISATION IN VIVO}

Sandrine Aspeslagh, ${ }^{1}$ Yali Li, ${ }^{2}$ Esther Dawen $\mathrm{Yu}_{1}{ }^{2}$ Nora Pauwels, ${ }^{3}$ Matthias Trappeniers, ${ }^{3}$ Enrico Girardi, ${ }^{2}$ Tine Decruy, ${ }^{1}$ Katrien Van Beneden, ${ }^{1}$ Koen Venken, ${ }^{1}$ Michael Drennan, ${ }^{1}$ Luc Leybaert, ${ }^{4}$ Jing Wang, ${ }^{2}$ Serge Van Calenbergh, ${ }^{3}$ Dirk M. Zajonc, ${ }^{2}$ Dirk Elewaut ${ }^{1}$ Laboratory for Molecular Immunology and Inflammation, Department of Rheumatology, Faculty of Medicine and Health Sciences, Ghent University, Ghent, Belgium.; 'Division of Cell Biology, La Jolla Institute for Allergy and Immunology, La Jolla, CA 92037, USA; ${ }^{2}$ Laboratory for Medicinal Chemistry, Faculty of Pharmaceutical Sciences, Ghent University, Ghent, Belgium; ${ }^{4}$ Department of Basic Medical Sciences - Physiology Group, Faculty of Medicine and Health Sciences, Ghent University, Ghent, Belgium

\subsection{6/ard.2010.148981.26}

Background and objectives Invariant natural killer T (iNKT) cells are a subset of highly conserved regulatory cells with features of innate and adaptive immunity. They possess a wide range of effector and regulatory activities due to their ability to produce copious amounts of effector cytokines. The authors have previously highlighted their regulatory role in experimental models of rheumatoid arthritis and spondyloarthritis. $\alpha$-Galactosylceramide is a prototype of iNKT cell agonist that, however, simultaneously leads to potent secretion of Th1, Th2 and Th17 cytokines. Therefore, optimising structure-function by chemical modification of $\alpha$-galactosylceramide to induce polarised Th responses is an attractive strategy for immune modulation.

Materials and methods Synthetic glycolipids were generated by modification of at the 6"- $0 \mathrm{H}$ position of galactose by addition of aromatic moieties, the only hydroxyl group not involved in hydrogen bond formation with the iNKT T cell receptor, suggesting the possibility of introducing modifications without the loss of important interactions. iNKT cell responses were measured by cytokine release in serum, BrdU incorporation and CFSE dilution. Calcium level analysis was performed by the calcium-sensitive probe Fura-2, epifluorescence microscopy and digital imaging. To evaluate the in vivo impact of Th1 bias, protection against murine B16 melanoma was studied. Surface Plasmon Resonance (SPR) studies were conducted using a Biacore 3000 (Biacore). Crystallisation and structure determination of CD1d-glycolipid-iNKT cell $\mathrm{T}$ cell receptor complexes were performed.

Results Structural analogs of $\alpha$-galactosylceramide obtained by derivatisation of the C-6"-position of galactose ring with selected aryl moieties, combine a marked Th1 polarised cytokine pattern, which is highly conserved between mice and men, with a marked tumour protection in vivo. Crystallographic data show that the strength of the Th1 response correlates well with the particular binding mode of these derivatives to CD1d. Surprisingly, the authors found that CD1d can undergo a conformational change in the A' roof upon lipid binding. Here, the modification of CD1d appears to be highly dependent upon the length of the linker connecting the aromatic moiety to the galactose, which results in the formation of an additional narrow and hydrophobic pocket. This structural modification is in striking contrast to the crystal structure of another Th1 biasing analog, $\alpha$-CGalCer, whose interaction follows a conventional key-lock principle.

Conclusion These findings highlight the previously unexploited flexibility of CD1d in accommodating galactosemodified glycolipids. The authors therefore speculate that these glycolipids may be useful targets for inducing, superior and more sustained iNKT cell responses in vivo. 